\title{
Nível de estresse em enfermeiros de duas instituições públicas do interior do Paraná
}

\author{
Stress level in nurses from two public institutions in the \\ interior of Paraná
}

Marcela Gonçalves Trevisan'; Jéssica Tainara Lopes de Jesus², Géssica Tuani Teixeira ${ }^{3}$, Lediana Dalla Costa ${ }^{4}$, Alessandro Rodrigues Perondi ${ }^{5}$

1. ORCID: http://orcid.org/0000-0002-1703-7200. Enfermeira. Especialista em Saúde Pública com Ênfase na Atenção à Saúde da Mulher. Docente do Curso de Graduação em Enfermagem da Universidade Paranaense (UNIPAR), Francisco Beltrão, PR, Brasil.

E-mail: marcelatrevisan@unipar.br

2. ORCID: http://orcid.org/0000-0002-2877-3254. Graduanda em Enfermagem pela Universidade Paranaense (UNIPAR), Francisco Beltrão, PR, Brasil.

E-mail: jessica.jesus@edu.unipar.br

3. ORCID: http://orcid.org/0000-0002-4479-1452. Enfermeira. Especialista em Saúde Pública com Ênfase na Atenção à Saúde da Mulher. Docente do Curso de Graduação em Enfermagem da Universidade Paranaense (UNIPAR), Francisco Beltrão, PR, Brasil.

E-mail: gessicateixeira@prof.unipar.br

4. ORCID: http://orcid.org/0000-0002-9114-3669. Enfermeira. Mestre em Saúde e Gestão do Trabalho. Docente e Coordenadora do Curso de Graduação em Enfermagem da Universidade Paranaense (UNIPAR), Francisco Beltrão, PR, Brasil.

E-mail: lediana@prof.unipar.br

5. ORCID: http://orcid.org/0000-0002-2001-8828. Enfermeiro. Mestre em Saúde e Gestão do Trabalho. Docente e Coordenadora do Curso de Graduação em Enfermagem da Universidade Paranaense (UNIPAR), Francisco Beltrão, PR, Brasil.

E-mail: alessandroperondi@prof.unipar.br

CONTATO: Autor correspondente: Marcela Gonçalves Trevisan | Endereço: Av. Júlio Assis Cavalheiro, 2000, Industrial. Francisco Beltrão, Paraná, Brasil.

E-mail: marcelatrevisan@unipar.br 


\section{RESUMO}

O estresse ocupacional exerce influência devastadora sobre a saúde do trabalhador. Sem dúvida, a enfermagem destaca-se entre as profissões consideradas estressantes. Logo, o objetivo foi identificar o nível de estresse em enfermeiros de duas instituições públicas de saúde do interior do Paraná. Trata-se de uma pesquisa exploratória-descritiva, com abordagem quantitativa, desenvolvida entre julho e agosto de 2020, com utilização da Escala Bianchi de Stress. Os dados foram submetidos à análise estatística descritiva. Entre os 17 enfermeiros que participaram no período estudado, identificou-se que o domínio D (Assistência de enfermagem prestada ao paciente) apresentou maior nível de estresse, com escore 4,4. Quando calculado o estresse geral, obteve-se o escore 3,7, indicativo de nível médio de estresse, o que corresponde a $64,7 \%$ dos enfermeiros. Desta forma, conclui-se que o nível de estresse se manteve médio e faz-se importante a implementação de medidas que minimizem a possível geração de fatores estressores entre os enfermeiros.

DESCRITORES: Estresse Ocupacional. Saúde Mental. Enfermagem.

\section{ABSTRACT}

Occupational stress has a devastating influence on the worker's health. Without a doubt, nursing stands out among the professions considered stressful. Thus, the objective was to identify the level of stress in nurses of two public health institutions in the interior of Paraná. It is a field research, exploratory-descriptive, of transversal character, with quantitative approach, developed in two units of reference, using a validated form, called Stress Bianchi Scale. The data were submitted to descriptive statistical analysis. Seventeen nurses participated in the study. It was identified that domain D presented higher stress level, with score 4.4. When the general stress was calculated, the score 3.7 was obtained, indicating the average stress level, which corresponds to $64.7 \%$ of the nurses. Thus, it was concluded that the stress level remained average. Thus, it is important to implement measures that minimize the possible generation of stress factors among nurses.

DESCRIPTORS: Occupational stress. Mental Health. Nursing. 


\section{INTRODUÇÃO}

O

estresse é um fator fisiológico definido pela excitação do estado emocional que

é manifestado pelo corpo humano em reação aos estímulos externos ${ }^{1}$. O termo estresse descreve o esforço exercido pelo organismo para se reorganizar frente aos estímulos hostis, considerados estressores, ou seja, é uma reação de luta ou fuga objetivando a homeostase ${ }^{2}$.

Quando o indivíduo é submetido a situações estressoras, o organismo desencadeia uma série de alterações neuroendócrinas que podem resultar no aumento da frequência cardíaca, hipertensão, tensão muscular, fadiga, irritabilidade, insônia, entre outros. Salienta-se que, a intensificação dos estímulos nocivos está intimamente relacionada à queda do rendimento geral e ao prejuízo das condições de saúde ${ }^{2}$.

No trabalho, denomina-se estresse ocupacional e é determinado pela incapacidade de tolerância, superação ou adaptação às exigências de natureza psíquica e que podem acarretar a diminuição da capacidade laboral ${ }^{1}$.

Considerado um grande problema da atualidade, o estresse ocupacional exerce influência devastadora sobre a saúde do trabalhador. Sem dúvida, a enfermagem destaca-se entre as profissões consideradas estressantes, ao passo que estes profissionais são afetados diretamente, constituindo-se agravo potencial de grande intensidade. Tal problemática merece uma atenção especial, visto que pode desencadear sérios problemas na saúde dos enfermeiros ${ }^{3}$.

Os enfermeiros são responsáveis pelas atividades de coordenação e supervisão do serviço, desenvolvem atividades de previsão, provisão de recursos materiais, dimensionamento de pessoal, liderança da equipe de trabalho, coordenação do processo assistencial e execução de procedimentos exclusivos de enfermagem e assumem posição de destaque na equipe de saúde, com desenvolvimento de estratégias para qualidade do cuidado 4 .

As pesquisas mostram que o atendimento a pacientes em unidade de urgência e emergência expõe esses profissionais a situações extremamente estressantes e desgastantes, pois, além de lidar com o sofrimento e morte, trabalham, normalmente, em condições longe de serem ideais com alta rotatividade de pacientes, imposto principalmente pelo elevado número de acidentes e de violência urbana que 
culminam, na maioria das vezes, com pessoas em estado crítico, além da carência de profissionais e de reconhecimento por gestores ${ }^{5-6}$.

Um estudo representativo realizado em Teresina - PI, identificou que os sinais mais comuns apresentados pelos enfermeiros foram: baixo desempenho na realização das atividades diárias, desânimo, irritação frequente e absenteísmo. E os mais graves foram: depressão, síndrome metabólica, fadiga crônica, dificuldades de dormir, diabetes, síndrome do pânico, dentre outas. Contudo, todos os sintomas causados pelo estresse ocupacional são reversíveis e os enfermeiros que apresentarem quaisquer alterações podem ficar inteiramente curados se houver intervenção com medidas adequadas para o enfrentamento das situações estressantes ${ }^{4}$.

Partindo-se dessa premissa, o presente estudo buscou responder a seguinte pergunta: Qual o nível de estresse apresentado por enfermeiros atuantes em duas instituições públicas de saúde municipal?

Para tanto, o objetivo deste estudo foi identificar o nível de estresse de enfermeiros em duas instituições públicas de um município do interior do Paraná.

\section{MÉTODO}

Pesquisa de campo, exploratória-descritiva, de caráter transversal, com abordagem quantitativa, desenvolvida em duas unidades de referência de um município do interior do Paraná. Trata-se de uma Unidade de Pronto Atendimento (UPA) e um Centro de Saúde Pediátrico.

A UPA configura-se como componente pré-hospitalar, de complexidade intermediária para o município sede e outros sete municípios e, o Centro de Saúde Pediátrico é referência municipal para atendimentos de urgência e emergência pediátrica.

A amostragem deu-se por conveniência, sendo composta por 17 enfermeiros que exercem atividades gerenciais e/ou de assistência direta aos pacientes e que aceitaram participar, mediante assinatura do Termo de Consentimento Livre e Esclarecido (TCLE), independe da idade, sexo e turno de trabalho.

Foram excluídos os profissionais afastados do trabalho por doenças, férias e licenças ou aqueles que não aceitaram participar. Logo, do total de 25 profissionais, 08 enquadraram-se nos critérios de exclusão. 
A pesquisa foi submetida e aprovada pelo Comitê de Ética em Pesquisa Envolvendo Seres Humanos (CEPEH) da Universidade Paranaense (UNIPAR), sob o Parecer 4.053.824. Salienta-se que foram preservados todos os preceitos éticos e legais da Resolução no 466/2012 do Conselho Nacional de Saúde (CNS).

A coleta de dados sucedeu-se entre os meses de julho e agosto de 2020, realizada por meio de um questionário, o qual foi entregue diretamente aos profissionais. Para não prejudicar a dinâmica de trabalho, disponibilizou-se um prazo de 10 dias para o preenchimento. Salienta-se que, por tratar-se de um instrumento validado na literatura, não foi realizado teste piloto.

O questionário está dividido em duas partes: a primeira relaciona-se às características dos trabalhadores, composta de dados sociodemográficos, de estilo de vida e profissionais (sexo, idade, situação conjugal, tabagismo, etilismo, escolaridade, cargo ocupado, tempo de formação, tempo de trabalho na unidade, turno de trabalho, $\mathrm{n}^{\circ}$ de empregos, horas de trabalho). E a segunda parte, refere-se à avaliação do estresse no trabalho utilizando-se a Escala Bianchi de Stress, validada para o contexto brasileiro ${ }^{7}$.

Trata-se de um questionário autoaplicável, composto por 51 itens, que recebem uma pontuação com variação de 1 a 7 . Os itens são divididos em 6 domínios e são compostos por atividades que envolvem a assistência e o gerenciamento do cuidado:

A - Relacionamento com outras unidades e supervisores (itens 40 a 46, 50 e $51)$;

B - Atividades relacionadas ao funcionamento adequado da unidade (itens 1 a $6)$;

C - Atividades relacionadas à administração de pessoal (itens 7 a 9, 12 a 14);

D - Assistência de enfermagem prestada ao paciente (itens 16 a 30);

E - Coordenação das atividades da unidade (itens 10, 11, 15, 31, 32, 38, 39, 47);

$\mathrm{F}$ - Condições de trabalho para o desempenho das atividades do enfermeiro (itens 33 a 37, 48, 49).

Na verificação do nível de estresse, a média analisada foi calculada pelos itens que compõem cada domínio, ou seja, para o cálculo do escore médio real, somou-se todos os valores diferentes de zero selecionados (51 subtraído o número de itens selecionados com 0 ), que foram divididos pelo total apontado como realizado. E ainda, 
para identificar o domínio de maior estresse, obteve-se o escore médio para cada área de $A-F$.

Sequencialmente, os escores foram classificados em níveis de stress, segundo as categorias: menor ou igual a 3,0 (baixo nível de stress), entre 3,1 a 4,0 (médio nível de stress), entre 4,1 a 5,9 (alerta para alto nível de stress) e maior ou igual a 6,0 (alto nível de stress).

Os dados coletados foram transferidos para uma planilha do Excel e submetidos à análise estatística descritiva, por meio do programa Statistical Package for the Social Sciences (SPSS) versão 25.0.

\section{RESULTADOS}

Participaram do estudo 17 enfermeiros, e destes, 12 exerciam atividades na UPA e cinco (05) no Centro de Saúde Pediátrico. Após análise dos dados, constatouse que os enfermeiros pesquisados eram predominantemente do sexo feminino $(94,1 \%)$, solteiros $(41,2 \%)$, com média de idade de 35,12 anos, prevalecendo a faixa etária superior a 31 anos (70,6\%). Com relação aos dados de formação, a maioria possui especialização $(64,7 \%)$, com tempo de formação superior ou igual a 10 anos $(58,8 \%)$.

Referente à atuação profissional, a maioria dos pesquisados atua na Unidade de Pronto Atendimento (UPA) (70,6\%). Quanto ao tempo e ao turno de trabalho na instituição, para $64,7 \%$ foi menor ou igual a um (01) ano, com predomínio do período diurno, respectivamente. No que tange ao vínculo empregatício, 52,9\% dos participantes possuíam um único emprego, com carga horária de até 40h semanais (58,8\%). Acerca do estilo de vida, $88,2 \%$ negaram tabagismo conquanto, 58,8\% referiram consumir bebida alcoólica socialmente, como mostra a Tabela 1.

Tabela 1. Distribuição dos enfermeiros conforme dados sociodemográficos, ocupacionais e de estilo de vida $(\mathrm{N}=17)$.

\begin{tabular}{|c|c|c|}
\hline Variáveis & $\mathbf{n}$ & $(\%)$ \\
\hline Idade (anos) - Média \pm DP & \multicolumn{2}{|c|}{$35,12 \pm 8,93$} \\
\hline Faixa etária & & \\
\hline Até 30 anos & 5 & $(29,4)$ \\
\hline$\geq 31$ anos & 12 & $(70,6)$ \\
\hline Sexo & & \\
\hline Feminino & 16 & $(94,1)$ \\
\hline Masculino & 1 & $(5,9)$ \\
\hline
\end{tabular}

Estado civil 
Solteiro

Casado $\quad 6$

União Estável 3

Divorciado 1

Escolaridade

Graduação 5

Especialização 11

Mestrado 1

Tempo de Formação

$\leq 1$ ano

Entre 2 e 5 anos

Entre 6 e 9 anos

$\geq 10$ anos

Instituição de Trabalho

Unidade de Pronto Atendimento (UPA) 12

Centro de Saúde Pediátrico

Tempo de Trabalho nessa Instituição

$\leq 1$ ano

Entre 2 a 5 anos

Entre 6 a 9 anos

Turno de Trabalho

Diurno

Noturno

Ambos os turnos

Número de Empregos

1 emprego

Horas de Trabalho

Até 40h semanais

Entre 41 e $60 \mathrm{~h}$ semanais

$\geq 60 \mathrm{~h}$ semanais

Tabagista

Nenhum

Menos que uma carteira ao dia

Consumo de Álcool

Nenhum

Socialmente

Fonte: Elaborado pelos autores (2020)

Com relação à classificação do estresse de forma individual, pode-se constatar que a maioria dos enfermeiros apresentou nível médio de estresse (64,7\%), seguido de baixo nível (23,5\%). Apenas 11,8\% apresentaram alto nível. Ao avaliar-se o estresse dos enfermeiros por domínios, evidenciou-se que a maioria $(70,6 \%)$ revelou baixo nível de estresse no domínio A (Relacionamento com outras unidades e supervisores). Para o domínio B (funcionamento adequado na unidade) observou-se prevalência de baixo e médio nível, ambos com $41,1 \%$. 
Nos domínios correspondentes à atividade relacionada à administração de pessoal e coordenação das atividades da unidade, destaca-se médio nível de estresse $(64,7 \%)$ para as duas variáveis. Os domínios D e $\mathrm{F}$ também apresentaram em sua maioria, nível médio com 52,9\%. Ainda, salienta-se que o domínio $\mathrm{F}$ (Condições de trabalho para desempenho das atividades) foi o único conjunto de atividades que não revelou níveis altos de estresse (Tabela 2 ).

Tabela 2. Distribuição percentual dos enfermeiros de acordo com a classificação do estresse geral e para cada domínio da Escala Bianchi de Stress ( $N=17)$.

\begin{tabular}{|c|c|c|c|c|c|c|}
\hline \multirow{3}{*}{ Variáveis } & \multicolumn{6}{|c|}{ Classificação } \\
\hline & \multicolumn{2}{|c|}{ Nível baixo } & \multicolumn{2}{|c|}{ Nível médio } & \multicolumn{2}{|c|}{ Nível alto } \\
\hline & $\mathbf{n}$ & $(\%)$ & $\mathbf{n}$ & $(\%)$ & $\mathbf{n}$ & $(\%)$ \\
\hline Estresse Geral & 4 & $(23,5)$ & 11 & $(64,7)$ & 2 & $(11,8)$ \\
\hline $\begin{array}{l}\text { Domínio A - Relacionamento com outras } \\
\text { unidades e supervisores }\end{array}$ & 12 & $(70,6)$ & 3 & $(17,6)$ & 2 & $(11,8)$ \\
\hline $\begin{array}{l}\text { Domínio B- Atividades relacionadas ao } \\
\text { funcionamento adequado da unidade }\end{array}$ & 8 & $(47,1)$ & 8 & $(47,1)$ & 1 & $(5,9)$ \\
\hline $\begin{array}{l}\text { Domínio C- Atividade relacionada à } \\
\text { administração de pessoal }\end{array}$ & 4 & $(23,5)$ & 11 & $(64,7)$ & 2 & $(11,8)$ \\
\hline $\begin{array}{l}\text { Domínio D- Assistência de enfermagem } \\
\text { prestada ao paciente }\end{array}$ & 5 & $(29,4)$ & 9 & $(52,9)$ & 3 & $(17,6)$ \\
\hline $\begin{array}{l}\text { Domínio E- Coordenação das atividades } \\
\text { da unidade }\end{array}$ & 4 & $(23,5)$ & 11 & $(64,7)$ & 2 & $(11,8)$ \\
\hline $\begin{array}{l}\text { Domínio F- Condições de trabalho para } \\
\text { desempenho das atividades }\end{array}$ & 8 & $(47,1)$ & 9 & $(52,9)$ & - & - \\
\hline
\end{tabular}

Fonte: Elaborado pelos autores (2020)

Por sua vez, ao obter-se o escore médio de cada domínio, a partir dos escores mensurados pelas perguntas contidas na escala Bianchi de Stress, verificou-se que, o domínio $D$ apresentou o maior escore: 4,4. Ao passo que, ao analisar-se o valor total do nível de estresse dos enfermeiros, obteve-se escore 3,7 (nível médio de estresse).

Em relação ao relacionamento com outras unidades e supervisores (domínio A), verificou-se que, o relacionamento com admissão/alta de paciente, configurou-se como o item mais estressante informado (escore 3,8). Já no domínio B, nota-se que o estresse de maior frequência foi previsão de material a ser usado (escore 5,8 ), conquanto para as atividades relacionadas à administração de pessoas (domínio $\mathrm{C}$ ), tem-se como item mais apontado, controlar a equipe de enfermagem (escore 4,7).

Nas atividades relacionadas à assistência de enfermagem prestada ao paciente (domínio D) o estressor que se destacou foi enfrentar a morte do paciente (escore 5,5). No domínio E correspondente à coordenação das atividades da unidade, o item controlar a qualidade do cuidado (escore 5,0 ) ganhou destaque. No que diz 
respeito às condições de trabalho para desempenho das atividades (domínio F), identificou-se que os estressores realizar atividades burocráticas (escore 3,9) e participar de eventos científicos (escore 3,9) foram os itens de maior desgaste entre os enfermeiros, nesse domínio.

\section{DISCUSSÃO}

A enfermagem constitui-se como a maior força de trabalho nas instituições hospitalares e o trabalho executado por essa categoria profissional é fundamental, pois está presente 24 horas por dia nas instituições de saúde e devido à escassez de trabalhadores, tem ritmo de trabalho intensificado, com consequentemente desgaste ${ }^{8}$.

O número insuficiente desse profissional, associado a um ritmo acelerado de trabalho, jornadas excessivas, atividades com alto grau de dificuldade, resultam em altas cargas de estresse ocupacional, trazendo importantes consequências para a qualidade de vida ${ }^{9}$.

Dentre os profissionais elegíveis para participar do presente estudo, a amostra foi composta por 17 enfermeiros, 12 atuantes na UPA e 5 na Unidade Pediátrica. Destes, houve predominância para o sexo feminino, solteiros, com faixa etária superior a 31 anos. Resultados semelhantes foram encontrados em estudo realizado no setor de urgência e emergência de um hospital regional de médio porte, localizado no município de Apodi (RN), onde houve predominância para profissionais do sexo feminino (70\%), com faixa etária entre 20 e 40 anos $(60 \%)^{10}$.

Os traços femininos estão atrelados a construção histórica da enfermagem, possivelmente pelo fato de suas precursoras serem mulheres. Fato que embora sirva como inspiração para as demais profissionais, até os dias atuais, aos poucos vem se desconstruindo. Ademais, faz-se importante salientar que, o estresse em mulheres ocorre com maior frequência em detrimento da dupla jornada de trabalho, com a associação às tarefas rotineira do mundo feminino ${ }^{8,10}$.

Em relação à formação, a maioria dos enfermeiros estudados apresentavam especialização, com tempo de formação superior ou igual a 10 anos. Em estudo conduzido em um hospital filantrópico de médio porte na região centro-oeste do Rio Grande do Sul, demonstrou resultados distintos para o tempo de formação dos profissionais, onde $75 \%$ dos enfermeiros estudados apresentavam até 10 anos de formação ${ }^{8}$. 
Já em relação à pós-graduação, um estudo realizado com enfermeiro de um hospital filantrópico de médio porte apontou resultados superiores, pois $75 \%$ dos participantes possuíam ao menos um curso de pós-graduação ${ }^{8}$. Dados divergentes evidenciam que as especializações proporcionam maior segurança para o enfrentamento de situações de desgastes no trabalho ${ }^{11}$.

Quanto ao tempo e ao turno de trabalho na instituição, o estudo demonstrou maior prevalência para profissionais com vínculo instrucional de um ano a menos, com predomínio de trabalho no período diurno. Achados inferiores para turno de trabalho foi encontrado no estudo realizado em hospitais públicos do município de São Paulo (SP) em que $46 \%$ dos enfermeiros trabalhavam no período diurno ${ }^{12}$.

Enfermeiros que trabalham no período noturno, sofrem grandes perturbações no padrão de sono e repouso. Assim, a chance de trabalhadores que desempenham atividades laborais à noite apresentarem o estado mental comprometido é maior, ao passo que a maioria apresenta irritabilidade ${ }^{13}$.

Concernente ao tempo de trabalho, resultados inferiores foram observados em estudo realizado com 24 enfermeiros de uma instituição hospitalar privada, localizada em um município da região oeste do Paraná, onde se identificou que $45,8 \%$ dos enfermeiros trabalhavam na instituição a um período menor ou igual a um ano ${ }^{14}$. $\mathrm{O}$ profissional que trabalha por um período maior na instituição, possui um controle melhor, por conhecer a instituição e sua rotina, pois sabe como desempenhar as atividades e estabelecer prioridades. Esse conjunto de fatores contribui para que 0 estresse seja menor ${ }^{15}$.

No que tange ao vínculo empregatício, a maioria dos participantes possuía um único emprego, com carga horaria de até $40 \mathrm{~h}$ semanais. Achado superior foi relatado na pesquisa sobre a relação da carga horária de trabalho e as reações de estresse de enfermeiros, onde constatou-se que 30,5\% realizavam 36 horas semanais e $69,5 \%$ realizavam mais de 36 horas semanais ${ }^{16}$. Comparativamente, ambos os estudos, apresentam profissionais com excesso de carga horária estipulada pelo Conselho Federal de Enfermagem (COFEN). A Resolução COFEN no 293/2004 regulamenta a elaboração da escala mensal dos profissionais de enfermagem, a qual deve corresponder a 36 horas semanais para atividades assistenciais e de 40 horas semanais para atividades administrativas.

O esgotamento profissional é visto como uma reação de tensão emocional crônica a partir do estresse excessivo no ambiente de trabalho, o que repercute na 
saúde física e mental do trabalhador, bem como, prejudica a concentração, a vigilância e a capacidade de supervisão ${ }^{3}$. Ao passo que, o estresse decorrente desse contexto representa um risco ao trabalhador de enfermagem, pois pode levar ao adoecimento, interferir diretamente na qualidade da assistência prestada e na própria qualidade de vida do profissional ${ }^{17}$.

Ao avaliar-se os domínios, observou-se que o domínio de maior estresse foi o domínio D - "Assistência de enfermagem prestada ao paciente", seguido do domínio C- "Atividades relacionadas à administração de pessoal" e domínio E - "Coordenação das atividades da unidade". O domínio D também se apresentou como o mais estressante em estudo realizado no município de Apodi (RN) em um hospital regional de médio porte ${ }^{10}$.

Semelhante aos achados do presente estudo, os domínios C e E foram apontados como os mais estressantes no estudo com enfermeiros no Rio Grande do Sul, sendo o domínio $C$ mais estressante, seguido pelo domínio $E^{8}$. Estudo com enfermeiros de emergência, alertou para alto nível de estresse com os maiores escores nos domínios referentes a atividades relacionadas à administração de pessoal e com exercício laboral de coordenação das atividades da unidade e assistência de enfermagem prestada ao paciente ${ }^{9}$.

No domínio $A$, correspondente às atividades de relacionamento com outras unidades e superiores, os enfermeiros foram identificados com baixos níveis de estresse. Entretanto, a atividade "Relacionamento com admissão/alta de paciente" configurou-se como o item mais estressante informado nesse domínio. A enfermagem enfrenta uma sobrecarga evidenciada pela responsabilidade em assumir mais que uma unidade hospitalar e potencializada pela complexidade das relações humanas envolvidas no processo de cuidado, sejam elas enfermeiro/cliente, enfermeiro/profissional de saúde e enfermeiro/familiares ${ }^{12}$. Desta forma, caso a instituição não ofereça a infraestrutura para o desempenho das atividades do enfermeiro, em qualquer área de atuação e, em especial nas unidades de internação, esse fator será estressante, uma vez que o profissional não poderá realizar com qualidade o trabalho.

As atividades de relacionamento com os profissionais de outros setores são necessárias para a continuidade da assistência ao paciente, de modo que acarreta desgaste físico, mental e social e, por conseguinte, sobrecarga de trabalho, o que reflete dificuldade em conciliar o gerenciamento da assistência com a prestação do 
cuidado ao paciente, acarretando na maioria das vezes, frustração no exercício das funções para o enfermeiro, fato esse, não demonstrado nas instituições estudadas ${ }^{9}$.

$O$ domínio $B$ refere-se às atividades relacionadas ao funcionamento adequado da unidade. No presente estudo, esse domínio demonstrou moderado a baixos níveis de estresse, sendo observado que a atividade mais estressante relatada pelos enfermeiros foi "Previsão de material a ser usado". O enfermeiro deve ter condições mínimas de material e pessoal para se dedicar a prestar uma assistência efetiva e eficaz diante das intercorrências, que são muito comuns nos setores de atendimento de urgência e emergência ${ }^{11}$. A falta de equipamentos e materiais para o adequado desempenho das atividades do enfermeiro gera, consequentemente, estresse no profissional de enfermagem, visto que não poderá realizar com qualidade o trabalho para o qual tem sua competência ${ }^{17}$.

$\mathrm{O}$ domínio $\mathrm{C}$, relativo à atividade relacionada à administração de pessoal despontou como um dos mais estressantes, e como parte desse domínio a situação "Controlar a equipe de enfermagem" obteve destaque. Um estudo com enfermeiros que desenvolviam atividades em unidades de emergência no município de Manaus/ $\mathrm{AM}$, demonstrou que o serviço gerencial do enfermeiro gerou alerta para alto nível de estresse ${ }^{9}$.

No entanto, sabe-se que o trabalho do enfermeiro para obter bons resultados depende da equipe técnica, o que pode ser considerado desafiador e levar ao estresse do profissional. Dessa forma, o controle da equipe pode ser avaliado como uma atividade estressora, devido à sobrecarga de trabalho, pois exige do enfermeiro habilidades como liderança e coordenação, além disso, demanda capacidade de relacionamento interpessoal.

Quanto ao domínio D, o item estressor "Enfrentar a morte do paciente" apresentou médio nível de estresse. No Rio Grande do Sul, um estudo apontou a mesma situação, sugerindo que pode estar associado ao fato de o profissional de enfermagem, desde a graduação apresentar dificuldades em lidar com pacientes terminais e, consequentemente, com a morte ${ }^{8}$.

No presente estudo, o domínio E - Coordenação das atividades da unidade, apresentou a terceira maior média de estresse, com o item "Controlar a qualidade do cuidado". Escores elevados em relação a controlar a qualidade do cuidado também foram encontrados em estudo realizado com enfermeiros de um hospital privado do Distrito Federal, corroborando os achados dessa pesquisa ${ }^{18 .}$ 
Os resultados obtidos no domínio $\mathrm{F}$ - Condições de trabalho para o desempenho das atividades do enfermeiro demonstraram que as situações de maior estresse foram "Participar de eventos científicos" e "Realizar atividades burocráticas", respectivamente. Realizar atividades burocráticas também foi citada como a atividade mais estressora nessa área ${ }^{19}$. A realização conjunta de atividades gerencias e assistenciais ampliam o desgaste e o sofrimento psicoemocional dos profissionais ${ }^{1}$.

\section{CONCLUSÃo}

Com base na aplicação da Escala Bianchi de Stress, conclui-se que os enfermeiros apresentaram nível médio de estresse. Ademais, o domínio de assistência de enfermagem prestada ao paciente foi considerado o mais estressante.

Sem dúvida, conhecer as atividades geradoras de estresse nos enfermeiros possibilitará a implementação de ações para prevenção e enfrentamento desta realidade, por meio da realização de programas de educação continuada, além da execução de ações contínuas de vigilância sobre a saúde psicológica destes trabalhadores, com vistas à redução desse agravo e à promoção do bem-estar biopsicossocial dos profissionais.

Desta forma, espera-se que os resultados desta pesquisa sirvam de subsídio para a chefias/equipes de enfermagem e administradores, a fim de minimizar os estressores apresentados.

Salienta-se, como limitação, a pequena população da amostra, o que confere baixo poder de generalização, com características bem específicas. Portanto, sugerese a realização de novas pesquisas com maior abrangência, principalmente ao considerar que, o estresse pode interferir na qualidade da assistência prestada ao paciente.

\section{REFERÊNCIAS}

1. Zavalis A, Paula VG, Machado DA, Marta CB, Perez Junior EF, Santiago LC. O nível de estresse dos enfermeiros na unidade de terapia intensiva. Rev. pesqui. cuid. fundam. [Internet]. 2019 [acesso em 2020 out 15];11(1):205-210. doi: https://doi.org/10.9789/2175-5361.2019.v11i1.205-210

2. Trettene AS, Ferreira JAF, Mutro MEG, Tabaquim MLM, Razera APR. Estresse em profissionais de enfermagem atuantes em unidades de pronto atendimento. Bol. Acad. Paul. Psicol. [Internet]. 2016 [acesso em 2020 set 02]; 
36(91):243-261 .

Disponível

em:

http://pepsic.bvsalud.org/scielo.php?script=sci arttext\&pid=S1415-

$\underline{711 \times 2016000200002}$

3. Santos WR, Silva RSPM, Souza SMA, Vitorio AMF. O esgotamento do profissional enfermeiro: influências na assistência à saúde. Rev. Cuidados em Saúde. [Internet]. 2017 [acesso em 2020 set 24];10(1). Disponível em: http://publicacoes.unigranrio.edu.br/index.php/rcs/article/view/4082/2239

4. Santana RS, Fontes FLL, Morais MJA, Costa GS, Silva RK, Araújo CS. et al. Occupational stress among emergency and urgent care nurses at a public hospital in Teresina, Piaui, Brazil. Rev. Bras. Med. Trab. [Internet]. 2019 [acesso em 2020 nov 03];17(1):76-82. doi: https://doi.org/10.5327/Z1679443520190295

5. Ferreira MM, Moura, $\mathrm{H}$. Enfermagem nas urgências e emergências: $\mathrm{O}$ estresse do profissional enfermeiro na unidade e atendimento de urgências e emergências, uma revisão bibliográfica. Rev. Terra e Cultura: Cadernos de Ensino e Pesquisa [Internet]. 2014 [acesso em 2020 out 14];30(58):27-38. Disponível em: http://periodicos.unifil.br/index.php/Revistateste/article/view/158

6. Teixeira GS, Silveira RCP, Mininel VA, Moraes JT, Ribeiro, IKS. Quality of life at work and occupational stress of nursing in an emergency care unit. Texto e Contexto Enferm. [Internet]. 2019 [acesso em 2020 nov 12];28:1-14. doi: https://doi.org/10.1590/1980-265x-tce-2018-0298

7. Bianchi ERF. Bianchi Stress Questionnaire. Rev. Esc. Enferm. USP. [Internet]. 2009 [acesso em set 23];43:1055-1062. doi: https://doi.org/10.1590/S0080$\underline{62342009000500009}$

8. Kirhhof RS, Marques OL, Susan B, Dias LLF, Squiavenato MCA. Nível de estresse entre enfermeiros de um hospital filantrópico de médio porte. Rev. Enferm. UFSM. [Internet]. 2016 [acesso em 2020 nov 5];6(1):29-39. doi: https://doi.org/10.5902/2179769217829

9. Fonseca JRF, Neto DL. Levels of occupational stress and stressful activities for nurses working in emergency. Rev. Rene. [Internet]. 2014 [acesso em 2020 out 10];15(5):732-742. doi: https://doi.org/10.15253/2175-6783.2014000500002

10. Freitas RJM, Lima ECA, Vieira ES, Feitosa RMM, Oliveira GYM, Andrade LV. Estresse do enfermeiro no setor de urgência e emergência. Rev. Enferm. UFPE on line. [Internet]. 2015 [acesso em 2020 out 25];9(10):1476-1483. Disponível em: https://periodicos.ufpe.br/revistas/revistaenfermagem/article/view/10861

11. Monte PF, Lima FET, Neves FMO, Studart RMB, Dantas RT. Stress among professional nurses working in intensive care units. Acta Paul. Enferm. [Internet]. 2013 [acesso em 2020 nov 11];26(5):421-427. doi: https://doi.org/10.1590/S010321002013000500004

12. Simonetti SH. Bianchi, ERF. Estresse do enfermeiro que atua em unidade de internação. Rev. Enferm. UFPE on line. [Internet]. 2016 [acesso em 2020 set 22];10(12):4539-4546. Disponível https://periodicos.ufpe.br/revistas/revistaenfermagem/article/view/11521/13411 
13. Montanholi LL, Tavares DMS, Oliveira GR. Estresse: fatores de risco no trabalho do enfermeiro hospitalar. Rev. Bras. Enferm. [Internet]. 2006 [acesso em 2020 out 15];59(5):661-5. doi: https://doi.org/10.1590/S0034-71672006000500013

14. Oliveira EB, Gallasch $\mathrm{CH}$, Junior PPAS, Oliveira AVR, Valério RL, Dias LBS. Estresse ocupacional e burnout em enfermeiros de um serviço de emergência: a organização do trabalho. Rev. Enferm. UERJ. [Internet]. 2018 [acesso em 2020 set 02];25:1-7. Disponível: https://www.epublicacoes.uerj.br/index.php/enfermagemuerj/article/view/28842

15. Silva AM, Guimarães LAM. Occupational Stress and Quality of Life in Nursing. Paidéia. [Internet]. 2016 [acesso em 2020 nov 04];26(63):63-70. doi: http://dx.doi.org/10.1590/1982-43272663201608

16. Dalri RCMB, Silva LA, Mendes AMOC, Robazzi MLCC. Nurses' workload and its relation with physiological stress reactions. Rev. Latino-Am. Enfermagem. [Internet]. 2014 [acesso em 2020 set 20];22(6):959-965. doi: http://dx.doi.org/10.1590/0104-1169.3292.2503

17. Almeida DM, Lopes LFD, Costa VMF, Santos RCTS, Corrêa JS. Avaliação do estresse ocupacional no cotidiano de policiais militares do Rio Grande do Sul. Organizações em contexto. [Internet]. 2017 [acesso em 2020 out 10];13(26):215138. Disponível em: https://www.metodista.br/revistas/revistasmetodista/index.php/OC/article/view/7206

18. Miranda, SMM. O nível de estresse do profissional de enfermagem que atua no centro cirúrgico em um hospital privado do Distrito Federal [Trabalho de Conclusão de Curso]. Brasília (DF): Faculdade de Ciências da Educação e Saúde FACES. 2018. [acesso em 2020 nov 11]. Disponível em: https://repositorio.uniceub.br/ispui/bitstream/235/11750/1/21396975.pdf

19. Ribeiro, KV. Estressores ocupacionais e níveis de estresse em enfermeiros de unidades de internação clínica. [Dissertação]. Rio de Janeiro (RJ): Universidade Federal do Estado do Rio de Janeiro. 2017. [acesso em 2020 set 15]. Disponível em: http://www.repositorio-bc.unirio.br:8080/xmlui/handle/unirio/10964 